\title{
REPLACING CONVEX SETS BY POLYTOPES
}

\author{
S. GALLIVAN ${ }^{1}$ AND J. ZAKS
}

ABSTRACT. A conjecture of A. J. Hoffman is settled by showing that if $P$ is a $d$-polytope in $E^{d}$, if compact convex subsets $C_{1}, \cdots, C_{k}$ are such that every $t$ dimensional affine flat that meets $P$ also meets $\mathbf{U}_{i=1}^{k} C_{i}$, then there exist polytopes $D_{1}, \cdots, D_{k}$, with $D_{i} \subseteq C_{i}$ for all $1 \leq i \leq k$, such that every $t$-flat that meets $P$ also meets $\mathbf{U}_{i=1}^{k} D_{i}$, provided $k \leq d-t+1$. Counterexamples are given for the cases where $d \geq 3,1 \leq t$ $\leq d-2$ and $k \geq d-t+2$.

A. J. Hoffman [4] proved that if $P$ is a $d$-polytope and if $C_{1}, \cdots, C_{k}$ are compact convex subsets of $P$, the union of which covers $P$, then there exist polytopes $D_{1}, \cdots, D_{k}$, with $D_{i} \subseteq C_{i}$ for all $1 \leq i \leq k$, such that $P=$ $\bigcup_{i=1}^{k} D_{i}$; he then stated the following

Conjecture $(d, t, k)$. If $P$ is a $d$-polytope in $E^{d}, C_{1}, \ldots, C_{k}$ are compact convex subsets of $P, t \geq 0$ is an integer such that every affine $t$-flat that meets $P$ also meets $\bigcup_{i=1}^{\bar{k}} C_{i}$, then there exist polytopes $D_{1}, \ldots, D_{k}$ with $D_{i} \subseteq C_{i}$ for all $1 \leq i \leq k$, such that every affine $t$-flat that meets $P$ also meets $\bigcup_{i=1}^{k} D_{i}$.

Obviously, Hoffman's theorem settles Conjecture $(d, 0, k)$ for all $d \geq 1$ and all $k \geq 1$. J. Zaks [6] gave counterexamples to Conjecture $(d, d-2,4)$, for all $d \geq 3$; this implies that Conjecture $(d, d-2, k)$ is false for all $d \geq 3$ and $k \geq 4$. J. Zaks [7] proved Conjecture $(d, d-1, k)$ for all $d \geq 2$ and $k \geq 0$; a simpler proof of this was given by H. G. Eggleston [1], where the dual form is treated. Conjecture $(d, t, 1)$ is clearly true, while Conjecture $(d, t, 2)$ was proved by W. R. Hare and C. R. Smith [3] for all $t$ satisfying $0 \leq t \leq d-1$.

The purpose of this paper is to prove Conjecture $(d, t, k)$ for all $d \geq 1$, all $t$ satisfying $1 \leq t<d-1$ and all $k$ satisfying $1 \leq k \leq d-t+1$; in addition, counterexamples will be given here to Conjecture $(d, t, d-t+2)$, for all $d \geq 3$ and all $t$ satisfying $1 \leq t \leq d-2$; this implies that Conjecture

Received by the editors October 30, 1973 and, in revised form, January 23, 1974. AMS (MOS) subject classifications (1970). Primary 52A25, 52A20.

Key words and phrases. $d$-polytope, affine flat, compact convex set.

${ }^{1}$ Research sponsored by British Science Research Council. 
$(d, t, k)$ is false for all $d \geq 3$, all $t$ satisfying $1 \leq t \leq d-2$ and all $k$ satisfying $k \geq d-t+2$. Consequently, Conjecture $(d, t, k)$ is settled for all $d, t$ and $k$.

The first author would like to thank D. G. Larman and P. McMullen for their invaluable assistance in the preparation of one of the versions of this paper.

Throughout the paper we shall follow the terminology of B. Grünbaum [2]; the $j$-skeleton of a polytope $P$, denoted by $\operatorname{skel}_{j} P$, is the union of all the faces of $P$ of dimension at most $j$.

We need the following few lemmas.

Lemma 1 (Compare with Lemma 1 of [3]). If $P$ is a d-polytope in $E^{d}$ and $C$ is a subset of $P$, such that every $t$-flat that meets $P$ also meets $C$, then skel ${ }_{d-t-1} P \subseteq C$.

Proof. Let $x \in \mathrm{skel}_{d-t-1} P$; there exists a hyperplane $H$ in $E^{d}$, such that $H \cap P$ is a face of $P$ of dimension at most $d-t-1$ which contains $x$. The dimension of $\operatorname{aff}(H \cap P)$ is at most $d-t-1$, hence $H$ contains a $t$-flat $L$, such that $L \cap \operatorname{aff}(H \cap P)=\{x\}$; hence $L \cap P=\{x\}$, and it follows from the condition of the lemma that $x \in C$, as required.

Lemma 2. If $P$ is a d-polytope in $E^{d}$, and $C_{1}, \ldots, C_{k}$ are compact convex subsets of $P$ such that every $t$ flat that meets $P$ also meets $\bigcup_{i=1}^{k} C_{i}$, and if $\bigcap_{i=1}^{k} C_{i} \neq \varnothing$, then there exist polytopes $D_{1}, \cdots, D_{k}$ with $D_{i} \subseteq C_{i}$ for all $1 \leq i \leq k$, such that every $t$-flat that meets $P$ also meets $\bigcup_{i=1}^{k} D_{i}$

Proof. Let $F_{1}, \cdots, F_{m}$ be the $(d-t-1)$-faces of $P$. It follows from Lemma 1 that skel ${ }_{d-t-1} P \subseteq \bigcup_{i=1}^{k} C_{i}$; hence $F_{j}$ is covered by $\bigcup_{i=1}^{k}\left(C_{i} \cap F_{j}\right)$. By Hoffman's theorem, applied to each one of the $(d-t-1)$-polytopes $F_{1}, \cdots, F_{m}$, it follows that there exist polytopes $D_{i j}$, for all $i$ and $j, 1 \leq$ $i \leq k$ and $1 \leq j \leq m$, such that

(1) $D_{i j} \subseteq C_{i} \cap F_{j}$ and

(2) $F_{j}=\bigcup_{i=1}^{k} D_{i j}$

Let $x \in \bigcap_{i=1}^{k} C_{i}$, and define $D_{i}$ by $D_{i}=\operatorname{conv}\left(\{x\} \cup \bigcup_{j=1}^{m} D_{i j}\right)$, for all $1 \leq i \leq k$. Clearly, $D_{i}$ is a polytope in $C_{i}$, for all $1 \leq i \leq k$;

Claim. Every $t$-flat that meets $P$ also meets $\bigcup_{i=1}^{k} D_{i}$.

To show this, let $L$ be a $t$-flat in $E^{d}$ that meets $P$, and let $M$ be a $(d-t)$-flat in $E^{d}$, orthogonal to $L$. Define the set $Q$ by $Q=\{\lambda x+(1-\lambda) y\}$ $y \in$ skel $\left._{d-t-1} P, 0 \leq \lambda \leq 1\right\}$; clearly $Q \subseteq \bigcup_{i=1}^{k} D_{i}$, hence it suffices to show that $L$ meets $Q$. 
Let $\operatorname{proj}_{M}: E^{d} \rightarrow M$ be the orthogonal projection of $E^{d}$ onto $M$; as is well known [5], see also $\left[2\right.$, p. 74, Theorem 8], $\operatorname{proj}_{M}(P)$ is a polytope of dimension $d-t(=\operatorname{dim} M)$, hence

$$
\text { rel bd } \operatorname{proj}_{M}(P) \subseteq \operatorname{proj}_{M}\left(\operatorname{skel}_{d-t-1} P\right) .
$$

Since $Q \supseteq$ skel $d_{-t-1} P$, it follows that $\operatorname{proj}_{M}(P)=\operatorname{proj}_{M}(Q) ; \operatorname{proj}_{M}(L)$ is a point, since $L \perp M$, and $\operatorname{proj}_{M}(L) \in \operatorname{proj}_{M}(P)$, because $L$ neets $P$; therefore $\operatorname{proj}_{M}(L) \in \operatorname{proj}_{M}(Q)$. It follows now, by taking the inverse of $\operatorname{proj}_{M}$, that $L$ meets $Q$.

This completes the proof of Lemma 2.

Lemma 3. If $C_{1}, \ldots, C_{d-t+1}$ are compact convex sets in $E^{d}$, such that $\cap_{i=1}^{d-t+1} C_{i}=\varnothing$, and if $0<t \leq d-1$ and every collection of $d-t$ of the sets $C_{1}, \ldots, C_{d-t+1}$ has a nonempty intersection, then there exists a $t$-flat $L$ in $E^{d}$ such that

(1) $L \cap\left(\bigcup_{i=1}^{d-t+1} C_{i}\right)=\varnothing$ and

(2) $L \cap \operatorname{conv}\left(\bigcup_{i=1}^{d-t+1} C_{i}\right) \neq \varnothing$.

Proof. The proof is by induction on $k=d-t+1$; in case $k=2$, it follows that $t=d-1$ and that the two compact convex sets $C_{1}$ and $C_{2}$ are disjoint; the required $t$-flat $L$ is any of the hyperplanes that strictly separates $C_{1}$ from $C_{2}$.

Assume inductively that the statement is true for $k-1(=d-t)$, for $k>2$, and let $C_{1}, \ldots, C_{d-t+1}$ be compact convex sets in $E^{d}$, as described in the lemma.

$C_{1}$ and $\bigcap_{i=2}^{d-t+1} C_{i}$ are two nonempty disjoint compact convex sets, hence there exists a hyperplane $H$ that strictly separates them, i.e. $C_{1} C$ $H^{-}$and $\bigcap_{i=2}^{d-t+1} C_{i} \subset H^{+}$, where $H^{-}$and $H^{+}$are the two open half-spaces determined by $H$.

Define $C_{i}^{*}$ by $C_{i}^{*}=C_{i} \cap H$ for all $2 \leq i \leq d-t+1$. Clearly, $C_{i}^{*}$ is a compact convex set in the hyperplane $H$, and since $\bigcap_{i=2}^{d-t+1} C_{i} \subset H^{+}$, it follows that $\bigcap_{i=2}^{d-t+1} C_{i}^{*}=\varnothing$.

Claim. Every collection of $d-t-1$ of the sets $C_{2}^{*}, \ldots, C_{d-t+1}^{*}$ has a nonempty intersection.

Let $T^{*}$ be such an intersection, and let $T$ be the corresponding intersection of the $d-t-1$ of the sets $C_{2}, \ldots, C_{d-t+1}$; clearly, $T^{*}=T \cap H$. $T \cap C_{1}$ is an intersection of $d-t$ of the sets $C_{1}, \ldots, C_{d-t+1}$, hence by the conditions of the lemma it follows that $T \cap C_{1} \neq \varnothing$. Therefore $T \neq \varnothing$; since $\bigcap_{i=2}^{d-t+1} C_{i} \subset H^{+}$and $T$ is the intersection of $d-t-1$ of the sets 
$C_{2}, \ldots, C_{d-t+1}$, it follows that $T \cap H^{+} \neq \varnothing$. Both $C_{1} \subset H^{-}$and $T \cap C_{1} \neq$ $\varnothing$ imply that $T \cap H^{-} \neq \varnothing$. Since $T$ is a convex set, it follows from both $T \cap H^{+} \neq \varnothing$ and $T \cap H^{-} \neq \varnothing$ that $T \cap H \neq \varnothing$; therefore $T^{*}=T \cap H \neq \varnothing$, and the proof of the claim is completed.

As a result, the inductive assumption is applicable to the sets $C_{2}^{*}, \ldots$, $C_{d-t+1}^{*}$ in the hyperplane $H$, implying the existence of a $t$-flat $L$ in $H$, such that

$$
L \cap\left(\bigcup_{i=2}^{d-t+1} C_{i}^{*}\right)=\varnothing \text { and } L \cap \operatorname{conv}\left(\bigcup_{i=2}^{d-t+1} C_{i}^{*}\right) \neq \varnothing .
$$

Since $C_{1} \subset H^{-}, L \subset H$ and $C_{i}^{*}=C_{i} \cap H$, it follows that $L \cap\left(\bigcup_{i=1}^{d-t+1} C_{i}\right)$ $=\varnothing$.

$L \cap \operatorname{conv}\left(\bigcup_{i=1}^{d-1+1} C_{i}\right) \supseteq L \cap \operatorname{conv}\left(\bigcup_{i=2}^{d-t+1} C_{i}\right) \supseteq L \cap \operatorname{conv}\left(\bigcup_{i=2}^{d-t+1} C_{i}^{*}\right) \neq \varnothing$ therefore $L \cap \operatorname{conv}\left(\bigcup_{i=1}^{d-t+1} C_{i}\right) \neq \varnothing$, which completes the proof of Lemma 3 .

Lemma 4. If $P$ is a polytope in $E^{d}$ and if $C_{1}, \ldots, C_{d-t+1}$ are compact convex subsets of $P$, such that

$$
P \neq \bigcup_{i=1}^{d-t+1} C_{i} \text { and } \bigcap_{i=1}^{d-t+1} C_{i}=\varnothing
$$

then there exists a toflat that meets $P$ and does not meet $\bigcup_{i=1}^{d-t+1} C_{i}$.

Proof. The proof is by induction on $k=d-t+1$. In case $k=2, t=$ $d-1$ and the two compact convex subsets $C_{1}$ and $C_{2}$ of $P$ are disjoint; if both of $C_{1}$ and $C_{2}$ are nonempty, then they are strictly separated by a hyperplane $H$, where $H$ meets $P$ and does not meet $C_{1} \cup C_{2}$, as required. If $C_{1}=\varnothing$ and $C_{2} \neq \varnothing$, then $P \neq C_{2}$ implies that at least one vertex $v$ of $P$ is not in $C_{2}$; any hyperplane that supports $P$ at $v$ has the property that it meets $P$ and does not meet $C_{1} \cup C_{2}$. The case where $C_{1}=C_{2}=\varnothing$ is trivial.

Assume inductively that the statement is true for $k-1, k>2$, and let $P$ be a polytope in $E^{d}, C_{1}, \ldots, C_{d-t+1}$ be compact convex subsets of $P$, where $d-t+1=k$, such that (1) is true.

If every collection of $d-t$ sets among the sets $C_{1}, \ldots, C_{d-t+1}$ has a nonempty intersection, then the result follows by Lemma 3. Otherwise, some $d-t$ of the sets among $C_{1}, \ldots, C_{d-t+1}$ have nonempty intersection, say $\bigcap_{i=1}^{d-t} C_{i} \neq \varnothing$. 
Since $P \neq \bigcup_{i=1}^{d-t+1} C_{i}$, let $x \in P \backslash \bigcup_{i=1}^{d-t+1} C_{i} ; x \notin \notin C_{d-t+1}$, hence there exists a hyperplane $H$ that contains $x$ and does not meet $C_{d-t+1}$. Define $P^{*}, C_{1}^{*}, \cdots, C_{d-t}^{*}$ by $P^{*}=P \cap H$ and $C_{i}^{*}=C_{i} \cap H$ for all $1 \leq i \leq d-t$. $P^{*}$ is a polytope in the $(d-1)$-flat $H, C_{1}^{*}, \ldots, C_{d-t}^{*}$ are compact convex subsets of $P^{*}$, such that

$$
P^{*} \neq \bigcup_{i=1}^{d-t} C_{i}^{*} \text { and } \bigcap_{i=1}^{d-t} C_{i}^{*}=\varnothing
$$

it follows by induction that $H$ contains a $t$-flat $L$ that meets $P^{*}$ and does not meet $\bigcup_{i=1}^{d-t} C_{i}^{*} L$ therefore does not meet $\bigcup_{i=1}^{d-t} C_{i}$, and since $H \cap$ $C_{d-t+1}=\varnothing$ and $L \subset H, L$ does not meet $C_{d-t+1}$; therefore $L \cap\left(\bigcup_{i=1}^{d-t+1} C_{i}\right)$ $=\varnothing$. Clearly $L$ meets $P$, since it meets $P^{*}$ and $P^{*} \subset P$.

This completes the proof of Lemma 4.

We are ready for the proof of our main result.

Theorem 1. If $P$ is a polytope in $E^{d}$ and $C_{1}, \ldots, C_{k}$ are compact convex subsets of $P$, such that every t-flat that meets $P$ also meets $\bigcup_{i=1}^{k} C_{i}$, and if $1 \leq t \leq d-2$ and $1 \leq k \leq d-t+1$, then there exist polytopes $D_{1}, \cdots, D_{k}$, with $D_{i} \subseteq C_{i}$ for all $1 \leq i \leq k$, such that every $t$-flat that meets $P$ also meets $\bigcup_{i=1}^{k} D_{i}$.

Theorem 1 states, of course, that Conjecture $(d, t, k)$ is true for all $d \geq 3,1 \leq t \leq d-2$ and $1 \leq k \leq d-t+1$.

Proof of Theorem 1. If $k<d-t+1$, then define $C_{i}=\varnothing$ for all $k<i$ $\leq d-t+1$. If $\bigcap_{i=1}^{d-t+1} C_{i} \neq \varnothing$, then the proof follows from Lemma 2 .

If $\bigcap_{i=1}^{d-t+1} C_{i}=\varnothing$, then since every $t$-flat that meets $P$ also meets $\bigcup_{i=1}^{d-t+1} C_{i}$. it follows from Lemma 4 that $P=\bigcup_{i=1}^{d-t+1} C_{i}$. There exist, by Hoffman's theorem, polytopes $D_{1}, \cdots, D_{d-t+1}$, with $D_{i} \subseteq C_{i}$ for all $1 \leq i \leq d-t+1$, such that $P=$ $\bigcup_{i=1}^{d-t+1} D_{i}$; it follows that every $t$-flat that meets $P$ meets $\bigcup_{i=1}^{d-t+1} D_{i}$, and the proof of Theorem 1 is complete.

As for the remaining cases of Conjecture $(d, t, k)$, we have

Theorem 2. Conjecture $(d, t, k)$ is false for all $d \geq 3,1 \leq t \leq d-2$ and $k \geq d-t+2$.

Proof. It is certainly sufficient to prove that Conjecture $(d, t, k)$ is false for all $d \geq 3,1 \leq t \leq d-2$ and $k=d-t+2$.

Case 1. $t=1$. The proof is by induction on $d$, starting with the counterexample to Conjecture $(3,1,4)$, given in [6]. 
Suppose $P$ is a polytope in $E^{d}$ and $C_{1}, \ldots, C_{d+1}$ are compact convex subsets of $P$, which provide a counterexample to Conjecture $(d, 1, d+1)$. Construct a counterexample to Conjecture $(d+1,1, d+2)$ as follows: regard $E^{d}$ as a subspace of $E^{d+1}$ in the usual way, take $x \in E^{d+1} \backslash$ aff $(P)$, and define

$$
\begin{aligned}
& P^{*}=\operatorname{conv}(\{x\} \cup P), \\
& C_{i=\operatorname{conv}}^{*}\left(\{x\} \cup C_{i}\right), \text { for all } i, 1 \leq i \leq d+1, \text { and } \\
& C_{d+2}^{*}=P .
\end{aligned}
$$

To show that $P^{*}, C_{1}^{*}, \ldots, C_{d+2}^{*}$ provide a counterexample to Conjecture $(d+1,1, d+2)$, let $L$ be any line in $E^{d+1}$ that meets $P^{*}$. If $L$ meets $x$ or $P$, then $L$ certainly meets $\bigcup_{i=1}^{d+2} C_{i}^{*}$. Otherwise, $L$ does not meet $\{x\} \cup P$; since $L$ meets $P^{*}, L$ meets some section of $P^{*}$ (= an intersection of $P^{*}$ with a hyperplane that strictly separates $x$ and $P$ ). This section of $P^{*}$, together with the corresponding sections of $C_{1}^{*}, \ldots, C_{d+1}^{*}$, is projectively equivalent to the original counterexample to Conjecture $(d, 1, d+1)$; therefore $L$ meets the union of the sections of $C_{1}^{*}, \ldots, C_{d+1}^{*}$, hence $L$ meets $\bigcup_{i=1}^{d+2} C_{i}^{*}$

Suppose $D_{1}^{*}, \cdots, D_{d+2}^{*}$ are polytopes, with $D_{i}^{*} \subseteq C_{i}^{*}$ for all $1 \leq i \leq d+$ 2. Let $H$ be a hyperplane, parallel to aff $(P)$, in $E^{d+1}$ that meets $P^{*}$ and misses $\{x\} \cup P$.

Considering the polytope $P^{*} \cap H$ with the collection of polytopal subsets $D_{1}^{*} \cap H, D_{2}^{*} \cap H, \ldots, D_{d+1}^{*} \cap H$ of $P^{*} \cap H$, and using the obvious projective equivalence of them with the original counterexample to Conjecture $(d, 1, d+1)$, it follows that $H$ contains a line $L$ that meets $P^{*}$ and does not meet $\bigcup_{i=1}^{d+1}\left(D_{i}^{*} \cap H\right)$. It follows that $L \cap P^{*} \neq \varnothing$ and $L \cap\left(\bigcup_{i=1}^{d+2} D_{i}\right)=$ $\varnothing$, hence $P^{*}, C_{1}^{*}, \cdots, C_{d+2}^{*}$ form a counterexample to Conjecture $(d+1$, $1, d+2)$; this completes the proof in Case 1 .

Case 2. $2 \leq t \leq d-2$. Let $P, C_{1}, \ldots, C_{d-t+2}$ in $E^{d-t+1}$ form a counterexample to Conjecture $(d-t+1,1, d-t+2)$, given by Case 1 of the proof, and let $P^{*}=P \times I^{t-1}, C_{i}^{*}=C_{i} \times I^{t-1}$ for all $1 \leq i \leq d-t+2$, where $I^{t-1}$ is the unit cube in $E^{t-1}$ and $A \times B$ denotes the Cartesian product of $A$ and $B$.

Let $L$ be a $t$-flat in $E^{d}$ that meets $P^{*} ; L \cap E^{d-t+1}$ is of dimension at least one, hence it (contains a line that) meets $\bigcup_{i=1}^{d-t+2} C_{i}$, therefore $L$ meets $\bigcup_{i=1}^{d-t+2} C_{i}^{*}$.

If $D_{1}^{*}, \ldots, D_{d-t+2}^{*}$ are polytopes in $E^{d}$, with $D_{i}^{*} \subseteq C_{i}^{*}$ for all $1 \leq i \leq$ $d-t+2$, then define $D_{1}, \cdots, D_{d-t+2}$ by taking $D_{i}$ as the image of $D_{i}^{*}$ under the orthogonal projection of $E^{d}$ onto aff $(P)$. Clearly $D_{i} \subseteq C_{i}$ for all 
$1 \leq i \leq d-t+2$. Therefore there is a line $L$ in aff $(P)=E^{d-t+1}$ that meets $P$ and does not meet $\bigcup_{i=1}^{d-t+2} D_{i}$, hence $L \times E^{t-1}$ is a $t$-flat in $E^{d}$ that meets $P^{*}$ and does not meet $\bigcup_{i=1}^{d-t+2} D_{i}^{*}$. Therefore $P^{*}, C_{1}^{*}, \ldots, C_{d-t+2}^{*}$ form a counterexample to Conjecture $(d, t, d-t+2)$.

This completes the proof of Theorem 2.

Summary. Hoffman's Conjecture $(d, t, k)$ was stated for all $d \geq 1,0 \leq$ $t \leq d-1$ and $k \geq 1$; the following table summarizes the complete solution to Conjecture $(d, t, k)$ :

\begin{tabular}{r|c|c|ll}
$d$ & $t$ & $k$ & Conjecture $(d, t, k)$ & by \\
\hline$\geq 1$ & 0 & $\geq 1$ & true & Hoffman [4]. \\
\hline$\geq 1$ & $>0$ & 1 & trivially true & \\
\hline$\geq 1$ & $>0$ & 2 & true & Hare-Smith [3]. \\
\hline 2 & $d-1$ & $\geq 2$ & true & Zaks [7]. \\
\hline$\geq 3$ & $d-2$ & $\geq 4$ & false & Zaks [6]. \\
\hline$\geq 3$ & $1 \leq t \leq d-2$ & $1 \leq k \leq d-t+1$ & true & Theorem 1, this paper. \\
\hline$\geq 3$ & $1 \leq t \leq d-2$ & $k \geq d-t+2$ & false & Theorem 2, this paper. \\
\hline
\end{tabular}

\section{REFERENCES}

1. H. G. Eggleston, Intersection of convex sets, J. London Math. Soc. (2) 5 (1972), 753-754. MR $47 \# 2493$.

2. B. Grünbaum, Convex polytopes, Pure and Appl. Math., vol. 16, Interscience, New York, 1967. MR 37 \# 2085.

3. W. R. Hare and C. R. Smith, On convex subsets of a polytope, Proc. Amer. Math. Soc. 35 (1972), 238-239. MR 46 \#789.

4. A. J. Hoffman, On the covering of polyhedra by polyhedra, Proc. Amer. Math. Soc. 23 (1969), 123-126. MR 40 \# 835.

5. V. Klee, Jr., Some characterizations of convex polyhedra, Acta Math. 102 (1959), 79-107. MR 21 \#390.

6. J. Zaks, On a conjecture of A. J. Hoffman, Proc. Amer. Math. Soc. 27 (1971), 122-125. MR 43 \# 1039.

7. — On a conjecture of A. J. Hoffman. II, Proc. Amer. Math. Soc. 34 (1972), 215-221. MR $45 \# 5869$.

DEP ARTMENT OF MATHEMATICS, UNIVERSITY COLLEGE, LONDON, ENGLAND DEP ARTMENT OF MATHEMATICS, UNIVERSITY OF HAIF A, HAIFA, ISRAEL

${ }^{2}$ This case is included in the last case of the table. 